\title{
Enhanced performance of fast-response 3-hole wedge probes for transonic flows in axial turbomachinery
}

\author{
D. Delhaye ${ }^{1}$, G. Paniagua ${ }^{1}$, J. M. Fernández Oro $^{2}$, R. Dénos ${ }^{3}$ \\ ${ }^{1}$ Turbomachinery and Propulsion Department, von Karman Institute for Fluid Dynamics. \\ Chaussée de Waterloo 72, B-1640 Rhode-Saint-Genèse, Belgium \\ paniagua@vki.ac.be \\ ${ }^{2}$ Área de Mecánica de Fluidos, Universidad de Oviedo. Campus de Viesques, E-33271 Gijón, \\ Spain \\ jesusfo@uniovi.es \\ ${ }^{3}$ Directorate General for Research, European Commission, CDMA/04-137, B-1049 Brussels, \\ Belgium
}

\begin{abstract}
This contribution presents the development, calibration, and application of a directional, three-sensor fast-response wedge probe for aerodynamic measurements in turbomachines. The probe is equipped with flush-mounted commercial fast-response miniaturized pressure transducers. Angular calibrations cover a wide range of Mach numbers $(0.3-0.7)$. The angular range of the probe and linearity of the calibration maps is enhanced with a novel zonal calibration technique. . The effect of the pressure readings uncertainty on the retrieved flow variables is analyzed for different Mach numbers, and the effect of self-induced perturbations is discussed. The effects of the Reynolds and Mach numbers are studied through spectral analysis of the calibration tests together with CFD simulations.

The data reduction methodology is explained and demonstrated with measurements performed in a single-stage high pressure turbine mounted in the compression tube facility of the von Karman Institute. The turbine was operated at subsonic and transonic pressure ratios (2.4 and 5.1) for a Reynolds number of $10^{6}$, representative of modern engine conditions. Time-resolved angular measurements were obtained at high speed conditions downstream of the rotor and allowed studying secondary flows and the rotor trailing edge shocks for different test conditions.
\end{abstract}

Keywords: Turbomachinery, directional probes, transonic flows

\section{Nomenclature}

\section{Roman symbols:}

$\begin{array}{lll}A & {\left[\mathrm{bar} / \mathrm{V}^{2}\right]} & \text { temperature sensitivity } \\ B & {[\mathrm{bar} / \mathrm{V}]} & \text { temperature offset } \\ C & {[\mathrm{bar} / \mathrm{V}]} & \text { temperature sensitivity } \\ C p & \left.\text { [deg. }^{-1}\right] & \text { Sensitivity coefficient } \\ C_{s, a x} & {[\mathrm{~m}]} & \text { Stator axial chord } \\ C_{r, a x} & {[\mathrm{~m}]} & \text { Rotor axial chord } \\ D & {[\mathrm{bar}]} & \text { temperature offset }\end{array}$




\begin{tabular}{|c|c|c|}
\hline$d$ & {$[\mathrm{~m}]$} & characteristic length \\
\hline$f$ & {$\left[\mathrm{~s}^{-1}\right]$} & frequency \\
\hline$k$ & {$[-]$} & conditioning coefficient \\
\hline$K_{\text {yaw }}$ & {$[-]$} & yaw angle coefficient \\
\hline$K_{\text {Mach }}$ & {$[-]$} & Mach number coefficient \\
\hline$l$ & {$[\mathrm{~mm}]$} & distance from sensor to apex (from the center of the sensor) \\
\hline$L$ & {$[\mathrm{~mm}]$} & length of wedge side \\
\hline$M$ & {$[-]$} & Mach number \\
\hline$P$ & [bar] & pressure \\
\hline $\operatorname{Re}$ & {$[-]$} & Reynolds number $=\rho U d / \mu$ \\
\hline$S t$ & {$[-]$} & Strouhal number $=\mathrm{f} \cdot \mathrm{d} / \mathrm{U}$ \\
\hline$t$ & {$[\mathrm{~s}]$} & Time \\
\hline$T$ & {$[\mathrm{~K}]$} & Temperature \\
\hline$T u$ & {$[\%]$} & Turbulence level \\
\hline$T_{r}$ & {$[\mathrm{~s}]$} & Rotor blade passing period \\
\hline$U$ & {$[\mathrm{~m} / \mathrm{s}]$} & velocity \\
\hline$V$ & {$[\mathrm{~V}]$} & voltage \\
\hline
\end{tabular}

\section{Greek symbols:}

$\begin{array}{lll}\alpha & {[\mathrm{bar} / \mathrm{V}]} & \text { pressure sensitivity } \\ \beta & {[\mathrm{bar}]} & \text { pressure offset } \\ \gamma & {[\mathrm{deg} .]} & \text { extent of calibration range } \\ \mu & {[\mathrm{kg} / \mathrm{m} . \mathrm{s}]} & \text { dynamic viscosity } \\ \varphi & {[\mathrm{deg}]} & \text { yaw angle } \\ \rho & {\left[\mathrm{kg} / \mathrm{m}^{3}\right]} & \text { density }\end{array}$

\section{Subscripts:}

$\begin{array}{ll}\infty & \text { far upstream } \\ 0 & \text { total value } \\ \text { atm } & \text { atmospheric } \\ S & \text { static value } \\ C & \text { central sensor } \\ L & \text { left sensor } \\ R & \text { right sensor } \\ r & \text { rotor frame of reference } \\ 1 & \text { stage inlet plane } \\ 3 & \text { stage outlet plane }\end{array}$

\section{Introduction}

Despite the advent of competing measuring techniques, directional pressure probes remain widely used in aerodynamic research because of their robustness and reliability. Their operation and data reduction are simpler than hotwire anemometry (Brunn, 1995), Laser Doppler Velocimetry (LDV) (Albrecht et al., 2003) and Particle Image Velocimetry (PIV) (Raffel et al., 2007). Pressure probes provide the velocity and pressure fields simultaneously and, unlike other sophisticated systems, have the added advantage of being compatible with data acquisition chains that can also be used for heat transfer sensors. Furthermore these probes are very versatile, allowing ad-hoc designs that can be better adapted to specific measurement conditions. 
The ongoing progress in miniaturization has led over the years to greater freedom in the design of fast-response probes by allowing mounting the sensors closer to the flow. Pressure probe designs can also be customized to allow easy replacement of damaged transducers, or to perform measurements in various locations along the probe. Moreover, the average time required to design, construct, calibrate, and set into operation a directional pressure probe is a handful of months, which is considerably less than the time needed to develop for example a PIV system in turbomachinery applications.

Directional probes can be operated in two different ways. In the yaw-null method, the probe is mounted on an actuator that rotates the probe until the pressure readings on the two opposite pressure taps are equal. The "average" flow angle can then be read directly. In the differential pressure method the probe is held stationary during the test and the angle is obtained from the differential pressure across the taps by a careful calibration at different incidence angles and Mach numbers. With this method, a large amount of data can be sampled in a short time and flow variations can be resolved up to the frequency response limit of the probe.

Pressure gauges can be mounted on the probe in two different ways, which will determine the probe frequency response. First, probes with sub-surface mounted sensors (capacitive, piezoresistive, or piezoelectric) maximize the spatial resolution, allowing minimum probe dimensions. This configuration also protects the sensors from aggressive conditions and allows cooling of the sensors (Zinn and Habermann, 2007). However the internal cavity underneath the measurement point and the capillary tube that transmits the pressure waves towards the sensor modifies the transfer function, introducing resonances that limit the bandwidth and may damp the high frequency fluctuations. A three-hole probe with sub-surface mounted sensors was investigated by Senoo et al. (1973) and Matsunaga et al. (1975) with frequency responses of about $1 \mathrm{kHz}$. The fidelity to measure unsteady flows with those remote-sensor probes was later studied by Dibelius and Minten (1983) and by Bohn and Schnittfeld (1992). More recently, a sub-surface mounted sensor design was developed by Sieverding et al., 2000, on a cylindrical/elliptical probe with trailing edge blowing for vortex street suppression.

Second, probes with flush-mounted sensors preserve the frequency response of the sensors (reaching above $200 \mathrm{kHz}$ when piezoresistive) while keeping the probe dimensions to a minimum. They are however more costly and prone to failure. The mounting of pressure transducers flush with the probe surface was pioneered by Kerrebrock et al. (1974) and Epstein (1985) using cylinder probes. Chip-on three-sensor wedge probes were subsequently developed by Bubeck and Wachter (1987), Cook (1989) and Cherret (1992). The mounting of bare piezo- 
resistive sensing elements directly on the probe was investigated at Oxford by Ainsworth et al. (1991) and at ETH Zurich by Gossweiler et al. (1990).

Choosing between sub-surface mounted or flush sensor designs has to be done in parallel with the choice of probe geometry and the number of sensors. The literature is profuse on diverse probe geometries and pressure taps arrangements. The number of sensors reflects a compromise between probe size and the number of flow variables retrieved. Wedge probes with flushmounted sensors prevent circulation effects related to the probe vortex shedding which is prominent in cylindrical probes. However recirculation bubbles appear close to the nose at high angles of incidence, compromising the dynamic response and the angular range. This problem is usually mitigated by placing the lateral holes downstream of the recirculation bubble. An extensive investigation of the respective effects of sensor location and wedge apex angle was conducted by Humm et al., 1994, and Ainsworth, 1995.

The frequency range of the probe is limited by the characteristic size of the probe (Lou et al., 1998), as well as the geometry and sensor implementation due to cavity resonances and sensor cut-off frequencies. Obtaining a high angular resolution typically requires to minimize the probe size; this can mean having holes in separated regions, which is detrimental on the probe frequency response.

The angular range of the probe depends on many factors, among which the location of the pressure taps is key (Humm et al., 1994), as well as their number. However, regardless of the probe performance, the angular range limitation is often imposed by the data reduction methodology. The literature provides a wide range of methods to perform the data reduction of pressure measurements. Dudzinsky and Kraus (1969) used graphical methods to obtain the angles and pressures from the calibration maps. Hufman at al. (1980) applied the slender theory to derive the flow field around the probe. Reichert and Wendt (1994) performed a 2-dimensional Taylor series decomposition of the calibration map. Two and three-dimensional Navier-Stokes solvers were used by several researchers to perform detailed calibration maps, verifying only a few points experimentally. Redionitis and Chrysanthakopoulus (1998) used neural networks to reconstruct the calibration map. Noteworthy to the present paper, the least-squares method of Hodson and Dambach (1999) does not require unambiguous pressure coefficients, which results in an extension of the angular range.

The current research presents a zonal calibration methodology that allows extending the angular range of wedge probes while maintaining the frequency range of a conventional calibration (in excess of $25 \mathrm{kHz}$ ). To illustrate the advantage of this novel methodology, it was applied to the data reduction of measurements downstream of a transonic turbine using the VKI high speed 
directional wedge probe. The results highlight an angular range and frequency response bandwidth sufficient to resolve the flow phenomena at the rotor blade passing frequency $(6.9 \mathrm{kHz})$. The unsteady measurement of the flow angle, and of the total and static pressure at the turbine outlet allows the clear identification of the rotor tip leakage and trailing edge shocks.

\section{Probe development}

\section{Geometry design}

It was chosen to use three sensors to retrieve the flow angle in the blade-to-blade plane ("yaw"), the total pressure, and the Mach number, while minimizing the probe head size to obtain a high spatial resolution and a low flow blockage. The wedge geometry was selected over cylindrical geometries for two main reasons. First, the wedge surfaces are well suited for flush-mounted commercial piezoelectric gauges. The chip-on design eliminates the need to use a transfer function to recover the unsteady pressures (Paniagua and Dénos, 2002). Second, Brouckaert et al. (1998) showed that cylindrical probes are particularly sensitive to unsteady wake induced perturbations. Moreover this geometry facilitates sensor repairs.

Rodriguez (1984) and Cook (1989) and Ainsworth (1995) demonstrated that the angular sensitivity of wedge probes depends much more on the distance of the pressure sensor from the apex than on the wedge angle. The choice of the wedge angle is then a compromise between four constraints:

- minimize the disturbance to the flow,

- minimize the distance between the front pressure sensor and the lateral sensors to optimize spatial resolution,

- maximize the robustness of the probe,

- maximize the sensitivity and calibration range (Humm et al., 1994).

An apex angle of $60 \mathrm{deg}$. was chosen in the present study as an appropriate compromise for the research conducted in the VKI test tube facility. The sides of the apex measure $3.5 \mathrm{~mm}$. Two fast-response Kulite ${ }^{\circledR}$ miniaturized pressure transducers (LQ-062-15A) are flush-mounted on the sides of the probe, close to the trailing edges (see Fig. 1). The distance from the sensing element to the apex 0.76 times the length of the probe side (Fig. 1-c). A third sensor (XCQ-062-25A) is enclosed in a front-protruding cylinder with a diameter of $1.65 \mathrm{~mm}$. The axis of the cylinder is radially displaced by $2.5 \mathrm{~mm}$ from the center of lateral sensors (see Fig. 1-a), to further decrease the dimensions of the probe and thus to increase its spatial resolution. This displacement does 
not pose a problem because the flow is essentially two-dimensional in the radial plane. This was demonstrated in a previous investigation using a directional five-hole probe in the same turbine stage (Paniagua et al., 2001). The absence of a pneumatic line or cavity between the sensors and the flow allows measurements at high frequency avoiding spurious resonance effects.

\section{Probe wake induced perturbations and recirculation bubble}

Ainsworth et al. (2000) identified large separations regions generated at the sharp rear angles of the probe, behind the lateral sensors. CFD simulations were conducted in the present investigation to study this detrimental effect on the probe.

Two-dimensional unsteady Navier-Stokes simulations were performed with Fluent ${ }^{\circledR}$ on the cross section of the probe (at the level of the lateral sensors, depicted in Fig. 1-c) to investigate the effect of the wake induced perturbations and other periodic phenomena. A segregated solver was used with a k-epsilon viscosity model. The time discretisation was second-order implicit with a time step of $10^{-6} \mathrm{~s}$. The unstructured triangular mesh of 150.000 elements was refined close the probe surface to capture the boundary layer.

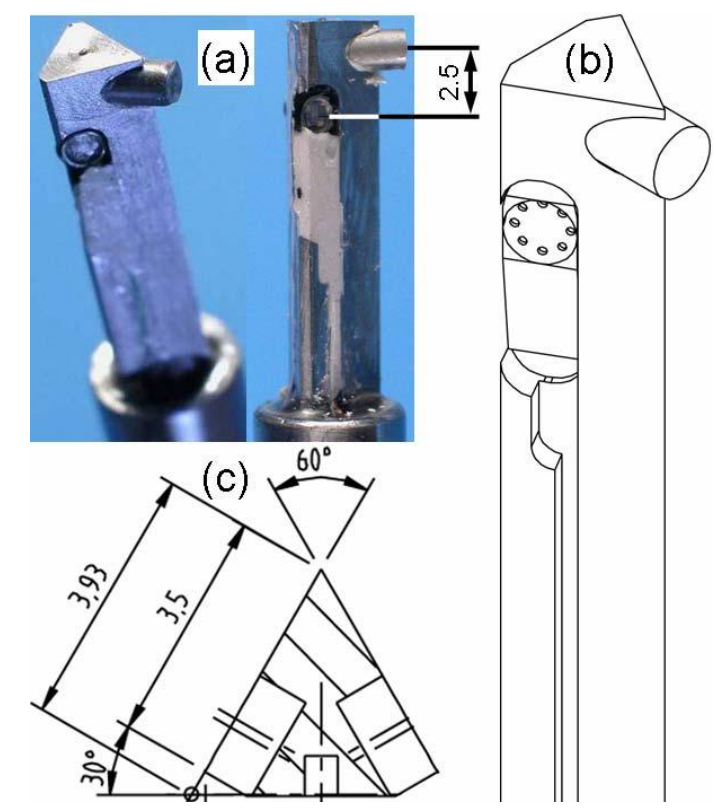

Fig. 1: Directional probe geometry. The cross-section cut is at the level of the lateral sensors.

Under the same conditions than that found in the calibration facility (Mach 0.7, atmospheric pressure), the iso-pressure lines show that the large separations are locked to the sharp rear corners of the probe (see Fig. 2). The numerical simulations show the presence of a recirculation bubble close to the apex of the probe when the probe is placed at a non-zero yaw angle. At a yaw 
angle of 20 deg., Fig. $2-a$, it is however of limited size: it extends from the apex to $l / L \approx 0.185$. At 30 deg., Fig. 2-b, the recirculation covers nearly the whole side of the probe $(l / L=0.87)$.

Vortices are shed alternatively from the two sides of the probe and induce pressure fluctuations on the lateral sensors. The effect of these fluctuations on the angular reading of the probe is influenced by their amplitude and relative phase. Table 1 summarizes the effect of the downstream vortex shedding for different angles. The decrease in vortex shedding frequency associated with the increase in angle is explained by the diminishing size of the area of the probe facing the flow orthogonally. Considering a characteristic dimension of $3.5 \mathrm{~mm}$, the Strouhal number at zero degree is 0.175 which is in-line with the literature (White, 2006). The maximum amplitude of the fluctuations occurs at $20 \mathrm{deg}$., resulting in a pulsation of pressure of nearly $20 \%$ of the local dynamic pressure.

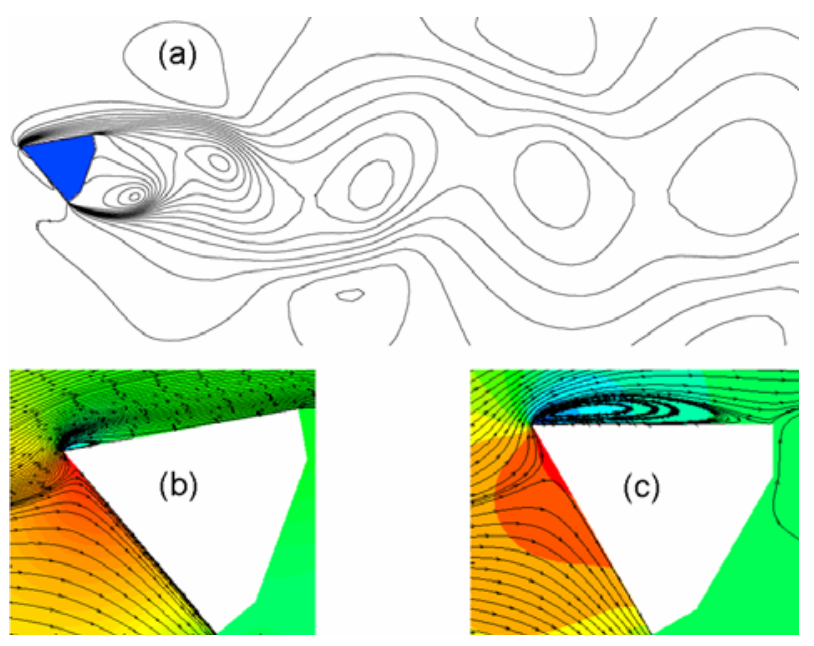

Fig. 2: Flow around the probe. The upstream flow is horizontal and goes from left to right. a) Total pressure contours at Mach 0.7 and a yaw angle of $20 \mathrm{deg}$. b) Streamlines showing the recirculation bubble at $20 \mathrm{deg}$. incidence (extending to $l / L \approx 0.185$ from the apex). c) Recirculation bubble at $30 \mathrm{deg}$. (extending to $l / L \approx 0.87$ from the apex)

Major interest is placed on the estimation of the influence of these lateral pressure fluctuations in terms of flow angle deviation and error of the Mach number. Previous evidences of the authors (with cylindrical probes, Argüelles et al, 2008) suggest that the increase the noise level at low frequencies is due to the separated conditions for the lateral sensors (i.e. the recirculation bubble in a wedge probe), rather than real effect of vortex shedding. On the contrary, wake-induced fluctuations occur at particular frequencies superimposed to other time scales of the turbulent flow that can be further removed (filtered) without significant loss of information. For conservatism, it is assumed in the present uncertainty analysis that the maximum fluctuation levels due to vortex shedding (19\% of the local dynamic pressure). The resulting error was estimated for the calibration at Mach 0.7 by adding these fluctuations to the lateral sensor 
readings and comparing the "perturbed" calibration with the "unperturbed" one. The results, included in Table 1, reveal a maximum error on the yaw angle of $2.3 \mathrm{deg}$. due to the large fluctuations on the right $\mathrm{Kulite}^{\circledR}$ sensor, and a maximum error on the Mach number of $7.5 \%$. Obviously, it is expected a notable reduction of these deviations in the regular operation of the probe, as recently confirmed in an experimental database of a single stage high pressure turbine mounted in the compression tube facility of the von Karman Institute (results shown later).

\begin{tabular}{||c|c|c|c|c|c||}
\hline \hline $\begin{array}{c}\text { Incidence } \\
\text { angle } \\
\text { [deg.] }\end{array}$ & $\begin{array}{c}\text { Dominant } \\
\text { frequency } \\
{[\mathrm{kHz}]}\end{array}$ & $\begin{array}{c}\text { Left pressure } \\
\text { fluctuations } \\
{\left[\mathrm{P} / \mathrm{P}_{\text {dynamic in } \%]}\right.}\end{array}$ & $\begin{array}{c}\text { Right pressure } \\
\text { fluctuations } \\
{\left[\mathrm{P} / \mathrm{P}_{\text {dynamic in } \%]}\right.}\end{array}$ & $\begin{array}{c}\text { Maximum Error in the } \\
\text { yaw angle } \\
\text { [deg.] }\end{array}$ & $\begin{array}{c}\text { Maximum Error in } \\
\text { the Mach number } \\
\text { [in \%] }\end{array}$ \\
\hline 0 & 11.5 & 8.0 & 8.0 & 0.53 & 5.4 \\
\hline 20 & 11.1 & 9.0 & 18.7 & 2.33 & 7.5 \\
\hline 30 & 9.2 & 2.8 & 11.0 & 1.25 & 4.6 \\
\hline
\end{tabular}

Table 1: Wake induced perturbations at Mach 0.7 as a function of the angle. Maximum errors in the yaw angle and Mach number due to vortex shedding.

\section{Influence of the Reynolds}

The effect of the Reynolds number on the probe readings is of particular importance because the calibration was performed at atmospheric pressure whereas the downstream static pressure in the turbine rig is roughly 0.5 bar. The sensitivity of the aerodynamic calibration to the Reynolds number was assessed through $2 \mathrm{D}$ numerical simulations. Both at atmospheric conditions $(\mathrm{Re}=$ $47000)$ and at turbine rig operation $(\mathrm{Re}=23000)$, the size of the recirculation bubble is nearly identical, it extends from apex to $l / L=0.158$ at $20 \mathrm{deg}$. yaw angle and $l / L=0.96$ at $30 \mathrm{deg}$. The pressure on the lateral sensors remains virtually unchanged for the two Reynolds numbers both at 0 and $30 \mathrm{deg}$. To compare both regimes, a non-dimensional pressure coefficient, denoted as $K_{\text {yaw }}=\left(\mathrm{P}_{\mathrm{L}}-\mathrm{P}_{\mathrm{R}}\right) /\left(\mathrm{kP}_{\mathrm{C}}-0.5\left(\mathrm{P}_{\mathrm{L}}-\mathrm{P}_{\mathrm{R}}\right)\right)$

obtained from a linear relationship between all the sensors readings, is shown in Fig. 3. More details will be given on the elaboration of the calibration curves in the next section. CFD calibration points have been also included in the plot. 


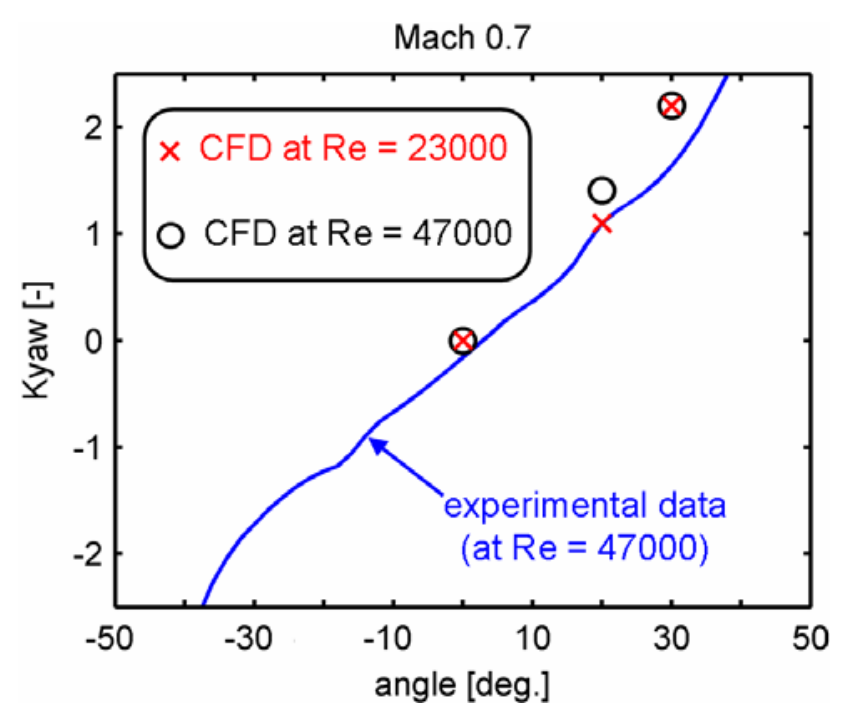

Fig. 3: $K_{\text {yaw }}$ calibration coefficient measured and predicted with CFD at two Reynolds number.

\section{Novel calibration methodology}

\section{Calibration bench}

The aerodynamic calibration of the probe was performed in a vertical-nozzle free-jet facility, illustrated in Fig. 4, which allows the calibration of directional probes up to the transonic domain. The air is coming from a 40 bar pressurized reservoir and enters into a settling chamber through a perforated cylinder. Several grids are installed in this section to control and guarantee the uniformity of the flow and the turbulence. The calibration nozzle is characterized by a contraction ratio of 14.75 and has an exit diameter equal to $50 \mathrm{~mm}$, allowing a stable operation, even at high Mach numbers. Important mass flows can be maintained during significant periods of time (up to a few minutes). Three different computerized carriages allow running a detailed angular (yaw and pitch) calibration. The accuracy of each step of the calibration is in excess of $+/-0.15 \mathrm{deg}$. The sampling frequency is set at $100 \mathrm{~Hz}$ while the probe rotates step by step. The angular calibration is performed as a function of the yaw angle, at Mach numbers spanning from 0.3 to 0.7 in increments of 0.1 . Acquisitions were performed from -50 to $50 \mathrm{deg}$. 


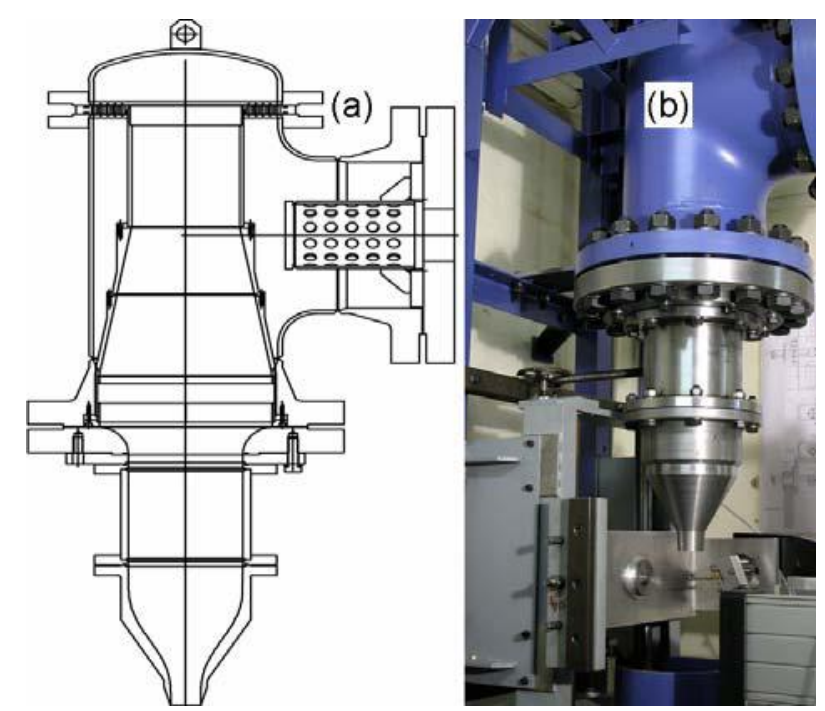

Fig. 4: Probe calibration wind tunnel.

\section{Correction of the transducer sensitivity to temperature}

The miniature Kulite ${ }^{\circledR}$ pressure transducers are composed of a thin silicon membrane on the surface of which piezoresistors are mounted (as displayed in Fig. 5-a,-b). The piezo-resistors are also sensitive to temperature variations. Hence a post-test numerical compensation must be applied when temperature transients occur. As sketched in Fig. 5-c the piezo-resistive gauge circuit delivers two voltages, one is an image of the uncompensated pressure on the membrane $\left(V_{\text {pressure }}\right)$ and the other an image of the temperature of the piezoresistors ( $\left.V_{\text {temperature }}\right)$. For each sensor, four coefficients are used in a bilinear law to recover the real pressure from the measured voltages:

$$
\begin{aligned}
& P=\alpha V_{\text {pressure }}+\beta \\
& \alpha=A V_{\text {temperature }}+B \\
& \beta=C V_{\text {temperature }}+D
\end{aligned}
$$

The coefficients are determined by an optimization routine which fits the sensor pressure under varying conditions of pressure and temperature to a low speed pneumatic probe recording. The optimization objective is the minimization of the sum of the absolute difference between the Kulite $^{\circledR}$ sensor and the pneumatic recording at all sampling points. This method is described in detail by Dénos, 2002. 

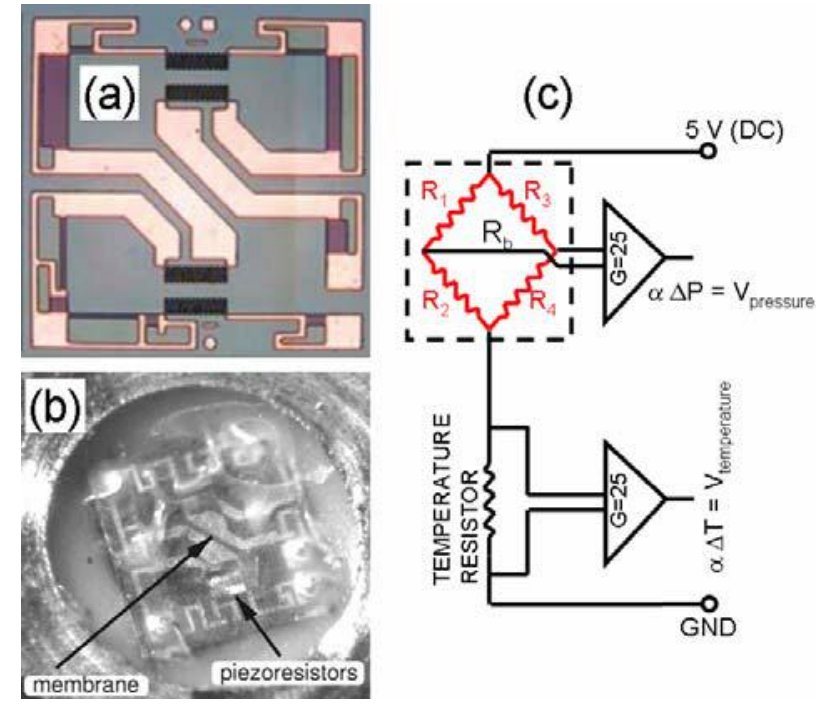

Fig. 5: a) Picture of a bare piezo-resistive gauge. b) Implementation of the Kulite gauge in a Pitot probe with a protective silicon layer. c) Active thermal compensation by the use $o$ a temperature resistor in series with the bridge

\section{Traditional data reduction}

The evaluation of each aerodynamic variable of interest requires the definition of an associated non-dimensional calibration coefficient. To retrieve the velocity vector as well as both the static and the total pressures, four coefficients are therefore chosen:

$$
\begin{gathered}
K_{\text {yaw }}=\frac{P_{L}-P_{R}}{k P_{C}-0.5\left(P_{L}+P_{R}\right)} \\
K_{\text {Mach }}=\frac{k P_{C}}{k P_{C}-0.5\left(P_{L}+P_{R}\right)} \\
K_{\text {tot }}=\frac{P_{0}-k P_{C}}{k P_{C}-0.5\left(P_{L}+P_{R}\right)} \\
K_{d y n}=\frac{P_{0}-P_{S}}{k P_{C}-0.5\left(P_{L}+P_{R}\right)}
\end{gathered}
$$

The coefficients are modified versions of the traditional ones proposed by Krause and Dudzinski (1969) $\left(K_{\text {yaw }}, K_{\text {tot }}\right.$ and $\left.K_{d y n}\right)$ and Brouckaert (2004) $\left(K_{\text {Mach }}\right)$. The modification consists in the addition of a constant multiplication factor $k=1.12$, to artificially increase the output of the central sensor. This factor is necessary for the calibration curves of the probe to follow a monotonous and quasi-linear evolution, and derives from the different shape of the pressure coefficient in the central protruding hole respect to the lateral ones. The main advantage of this set of coefficients is that both $K_{\text {yaw }}$ and $K_{\text {Mach }}$ are directly obtained from the sensors readings. From the calibration measurements, two-dimensional contour plots combining these two coefficients are created as displayed in Fig. 6 -left. Also, single maps are obtained for both $K_{\text {tot }}$ 
and $K_{d y n}$ separately. Note that this traditional definition gives a highly distorted map because of the strong gradients appearing in the definition of the coefficients near the edges of the domain, the map is rapidly diverted towards the limit where singular points arise, approx. $\pm 40 \mathrm{deg}$.

The algorithm used to retrieve the aerodynamic variables of the flow is as follows: for any given measurement, $K_{\text {yaw }}$ and $K_{\text {Mach }}$ are calculated according to (1). The algorithm then searches in the 2D contour plot (Fig. 6-left) to find corresponding cells in which both the experimental $K_{\text {yaw }}$ and $K_{\text {Mach }}$ are contained. A bilinear subgrid is then laid on the selected cells and the same process is repeated to improve the precision. The angle and Mach number values are finally taken as the mean values of the corresponding subcells. The total and static pressures are then retrieved directly from the other single contour plots, reading the value from the angle and Mach number inputs as usual. For this algorithm to yield a single value of angle and Mach number, the map of $K_{\text {yaw }}$ must be monotonous in the direction of increasing yaw, and the map of $K_{\text {Mach }}$ must be monotonous in the direction of increasing Mach.

\section{Zonal calibration}

Typically, traditional calibration is restricted by the emergence of singularities in the calibration coefficients when the denominator becomes zero (eq 1). This restriction reduces the effective angular range of this type of probes to a limited centered interval, with a span depending on the characteristics of every particular probe (geometry, construction angle...). Recently, Argüelles et al. (2008) have proposed the convenience of a zonal definition of the calibration coefficients in order to prevent the arising of singular points. This technique discriminates several zones for the angular range of the calibration, which are identified using the pressures measured in the holes. Thus, each zone corresponds to the angular interval where the pressure on one of the sensor is higher than on the others. The four coefficients of the traditional data reduction are therefore defined for three different zones, where the yaw and Mach coefficients are defined as follows:

$$
\begin{aligned}
& K_{\text {yaw }}=\frac{P_{L}-P_{R}}{k_{Z} P_{C}-0.5\left(P_{L}+P_{R}\right)} \quad K_{\text {Mach }}=\frac{0.25 k_{Z} P_{C}}{k_{Z} P_{C}-0.5\left(P_{L}+P_{R}\right)} \quad \text { if } k_{Z} P_{C}>P_{L}, P_{R} \\
& K_{\text {yaw }}=4+\frac{P_{R}-P_{L}}{P_{L}-0.5\left(k_{Z} P_{C}+P_{R}\right)} \quad K_{\text {Mach }}=\frac{k_{Z} P_{C}}{k_{Z} P_{C}+P_{L}-2 P_{R}} \quad \text { if } P_{L}>k_{Z} P_{C}, P_{R} \\
& K_{\text {yaw }}=4-\frac{P_{R}-P_{L}}{P_{R}-0.5\left(k_{Z} P_{C}+P_{L}\right)} \quad K_{\text {Mach }}=\frac{k_{Z} P_{C}}{k_{Z} P_{C}+P_{R}-2 P_{L}} \quad \text { if } P_{R}>P_{L}, k_{Z} P_{C}
\end{aligned}
$$

The definition adopted for the central zone is the traditional one whereas others expressions are used for the lateral zones. An iterative process was conducted to determine appropriate 
experimental coefficients in both the numerator and the denominator. With such expressions, it is possible to minimize the impact of the recirculation appearing for yaw angles greater than $20 \mathrm{deg}$, and obtain a monotonous map of $K_{\text {Mach }}$. Fig. 6-right shows the final calibration map obtained with the zonal method. Like in the traditional calibration, the map is not symmetrical respect to the $K_{\text {yaw }}$ coefficient, due to slight imperfections in the manufacturing process, but the difference between the two sides has been significantly reduced. A constant multiplication factor, now $k_{Z}=1.05$, was introduced to increase the output of the central sensor and enhance the zonal discrimination of the coefficients. In addition, $K_{t o t}$ and $K_{d y n}$ have been made nondimensional using the same zonal denominator introduced for the yaw coefficient (there is no need to define a zonal discrimination for these coefficients).

Because the zonal method only differs from the traditional calibration method by the definition of the calibration coefficients, the algorithm to retrieve the flow variables does not need to be modified.
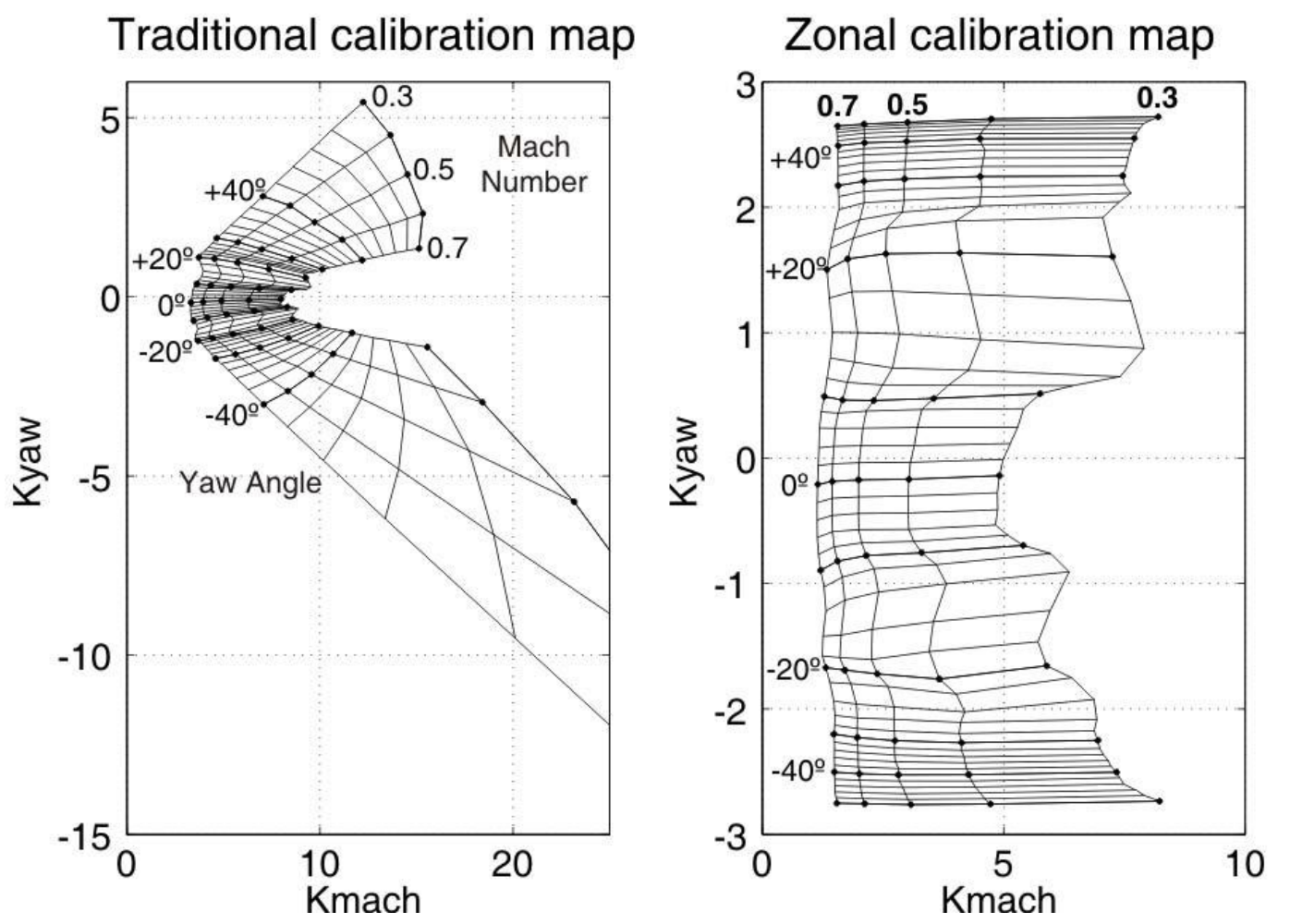

Fig. 6: Comparison of the calibration map using the conventional data reduction and the novel zonal calibration procedure.

\section{Optimized data reduction}

Two advantages of the zonal calibration method immediately emerge. First, the useful angular range extends to cover the whole range of the calibration. Second, the calibration maps become 
quasi-orthogonal, particularly at high Mach numbers (Fig. 6-right), thus decreasing the uncertainty in previously highly skewed regions of the calibration maps. This could potentially allow the calibration of the probe over an even wider range and at higher Mach numbers, because the flow angle and the local Mach number are determined identifying the cell on the map where both $K_{\text {Mach }}$ and $K_{\text {yaw }}$ coordinates collide.

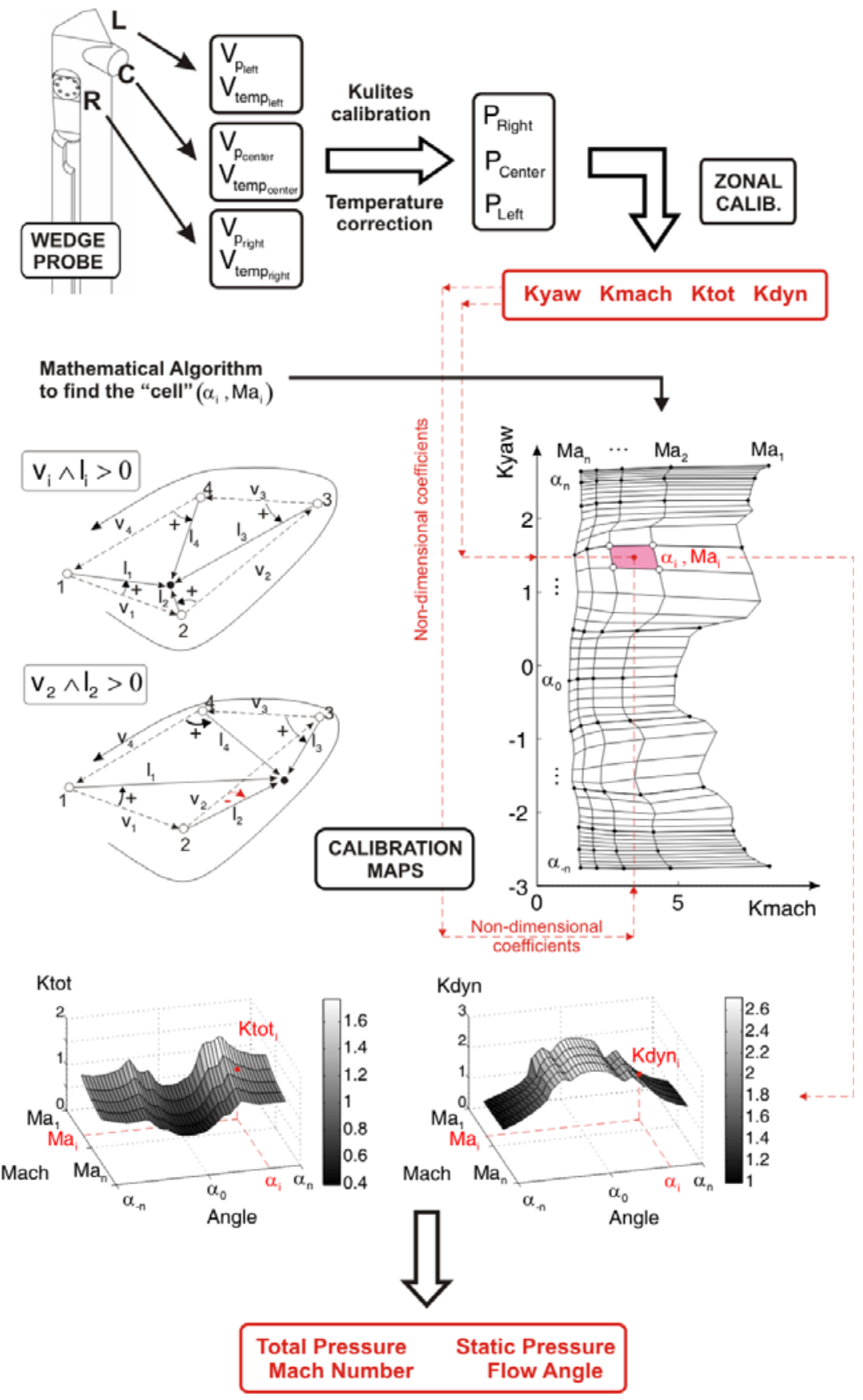

Fig. 7: Data processing flow chart.

Fig. 7 shows a detailed flowchart of the data processing employed during the operation of the wedge probe. The correction of the transducer sensitivity to temperature is first introduced to 
transform voltage outputs into pressure readings. Then the zonal method determines the primary variables of the flow (angle and Mach number) using a mathematical algorithm that finds the calibration square cell corresponding the local flow conditions. The algorithm, based on a vectorial criterion is outlined in the figure. The measured $K_{\text {Mach }}$ and $K_{\text {yaw }}$ are inside a calibration cell only when the four vectorial products $v_{i} \times l_{i}$ are bigger or equal than 0 (see Fig. 7). Successive bilinear interpolations are performed inside the cell to improve the accuracy. Finally, the pressure values are retrieved from the maps shown at the bottom of Fig. 7.

\section{Uncertainty in the calibration}

In this section, the impact of the uncertainty on the pressure sensor readings on the retrieved variables is estimated using the method proposed by Kline, 1953. Given the described flow retrieval methodology, the uncertainty associated to $K_{\text {Mach }}$ and $K_{\text {yaw }}$ must first be evaluated. The corresponding expression for the uncertainty of both coefficients yields:

$$
I_{K_{\text {yaw }}}=\sqrt{\sum_{i=1}^{N}\left(\frac{\partial K_{\text {yaw }}}{\partial x_{i}} I_{x_{i}}\right)^{2}} \quad I_{K_{\text {Mach }}}=\sqrt{\sum_{i=1}^{N}\left(\frac{\partial K_{\text {Mach }}}{\partial x_{i}} I_{x_{i}}\right)^{2}}
$$

where $x_{i}$ represents the three pressure readings. Applied to the zonal calibration method, we obtain:

$$
\begin{aligned}
& I_{K_{\text {yaw }}}=\frac{1}{Q_{\text {yaw }}} \sqrt{\left(k_{Z} K_{\text {yaw }}\right)^{2} I_{X C Q}^{2}+\left(2+\frac{K_{\text {yaw }}^{2}}{2}\right) I_{L Q}^{2}} \text { if } k_{Z} P_{C}>P_{L}, P_{R} \\
& I_{K_{\text {yaw }}}=\frac{1}{Q_{\text {yaw }}} \sqrt{\left(\frac{k_{Z}}{2} K_{\text {yaw }}\right)^{2} I_{X C Q}^{2}+\left(2+\frac{5\left(K_{\text {yaw }}-4\right)^{2}}{4}\right) I_{L Q}^{2}} \quad \text { if } k_{Z} P_{C}<P_{L}, P_{R} \\
& I_{K_{\text {Mach }}}=\frac{1}{Q_{\text {Mach }}} \sqrt{\left(\frac{1}{8}+K_{\text {Mach }}^{2}\right) k_{Z}^{2} I_{X C Q}^{2}+\frac{1}{2} K_{\text {Mach }}^{2} I_{L Q}^{2}} \quad \text { if } k_{Z} P_{C}>P_{L}, P_{R} \\
& I_{K_{\text {Mach }}}=\frac{1}{Q_{\text {Mach }}} \sqrt{\left(1+K_{\text {Mach }}^{2}\right) k_{Z}^{2} I_{X C Q}^{2}+5 K_{\text {Mach }}^{2} I_{L Q}^{2}} \text { if } k_{Z} P_{C}<P_{L}, P_{R}
\end{aligned}
$$


where $Q_{\text {yaw }}$ and $Q_{\text {Mach }}$ represent the denominators in the definition of the coefficients (eq. 3), and $I_{X C Q}, I_{L Q}$ are the uncertainty of the central and lateral Kulites ${ }^{\circledR}$. Outside the central region (where the pressure in the central sensor is predominant), the expression of the uncertainty is similar for the lateral regions. Introducing these uncertainties in the data reduction pipeline, it is now possible to estimate the uncertainty on the flow angle and the Mach number.

The uncertainty distribution on the Mach number and the yaw angle is plotted in Fig. 8 over the whole angular span of the calibration for Mach 0.4 and 0.6. The red lines correspond to the estimation of the uncertainty using the traditional calibration (the first expression in eq. 5 and eq. 6 is employed over the whole angular range), while black lines show the result of the zonal technique. Around zero yaw angle, the uncertainty is approximately $4 \%$ at Mach 0.4 , and $1.75 \%$ at Mach 0.6. The difference in the central zone between traditional and zonal techniques is due to the different multiplication factor employed for the central sensor. The uncertainty increases in the vicinity of $+/-40 \mathrm{deg}$, especially in the case of the traditional calibration method.
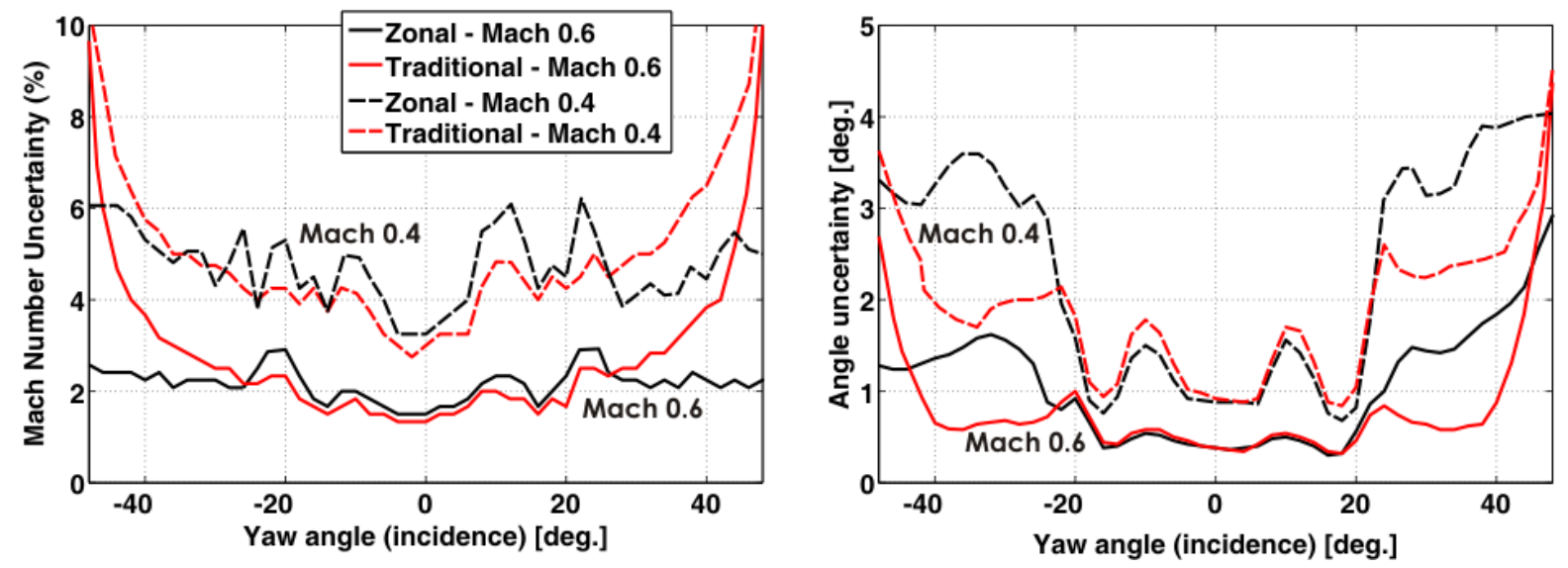

Fig. 8: Calibration uncertainties in the yaw angle and Mach number.

The angular calibration was conducted twice to estimate repeatability. The maximum total pressure difference was $1.15 \%$, but this was partly caused by a change in the temperature of the air from the calibration facility after successive runs.

\section{Results and discussion}

\section{Experimental apparatus: Turbine model and test rig}

The turbine stage is composed of uncooled cylindrical vanes and uncooled rotor leaned blades. The design characteristics of both the stator and rotor are summarized in Table 2. Fig. 9-a 
displays a view of the turbine stage, while Fig. 9-b depicts the measurement planes. The fastresponse 3-hole probe was placed at the rotor outlet (plane 3) at different spans.

The measurement campaign included two different pressure ratios, resulting in rotor relative outlet Mach numbers ranging from 0.65 to 1.18 . Table 3 summarizes the turbine operating conditions, including the stage loading and flow coefficient. In the following they will be referred to using the notation in the first column. The stage inlet conditions are identical for all tests, $\left(P_{01}=1.65 \mathrm{bar}, T_{01}=434 \mathrm{~K}\right.$, and $\left.T u \sim 5 \%\right)$. All tests were performed at $6500 \mathrm{RPM}$, i.e. $108.3 \mathrm{rev} / \mathrm{s}$, resulting in a blade passing frequencies of $6.9 \mathrm{kHz}$ respectively. Tests were performed with a corrected speed N/ $\sqrt{T_{01}}=311$ at a Reynolds number of $1.065 \times 10^{6}$ based on the vane outlet velocity and vane chord.

\begin{tabular}{||l|c|c||}
\hline & Vane & Rotor \\
\hline Number of airfoils & 43 & 64 \\
\hline Axial chord $\left(C_{a x}\right)[\mathrm{mm}]$ & 41.16 & 39.78 \\
\hline aspect ratio $\left(C_{a x} / h\right)$ & 0.812 & 0.738 \\
\hline $\mathrm{R}_{\text {hub }} / \mathrm{R}_{\text {tip }}$ & 0.872 & 0.864 \\
\hline stagger [deg.] & 54 & 32 \\
\hline pitch / $C_{a x}$ & 1.313 & 0.912 \\
\hline throat / pitch & 0.245 & 0.401 \\
\hline
\end{tabular}

Table 2: Vane and rotor design parameters.

\begin{tabular}{|c|c|c|c|c|c||}
\hline Condition & $\boldsymbol{P}_{01} / P_{\mathrm{s} 3}$ & $\boldsymbol{M}_{2, i \mathrm{~s}}$ & $\boldsymbol{M}_{3 r, i s}$ & $\Delta H / \mathrm{U}^{2}$ & $\boldsymbol{V}_{\mathrm{ax}} / \mathbf{U}$ \\
\hline Low & 2.42 & 1.071 & 0.65 & 1.28 & 0.48 \\
\hline High & 5.12 & 1.249 & 1.18 & 2.02 & 0.70 \\
\hline
\end{tabular}

Table 3: Turbine operating conditions.

The turbine measurements were performed in the von Karman Institute compression tube turbine test rig. The test section is fed with air by a compression tube, the outlet is connected to a dump tank by a duct equipped with a sonic throat which allows the adjustment of the downstream static pressure. This setup is capable of simulating the operating Reynolds numbers, pressure and temperature ratios found in actual gas turbines.

The output signal of the probe was split in two: an unamplified low pass filtered signal (at 750 $\mathrm{Hz}$ ), and an amplified high pass filtered signal (at $100 \mathrm{~Hz}$ ) sampled at $300 \mathrm{kHz}$. The first was used for time-averaged measurements and the second for time-resolved measurements. 

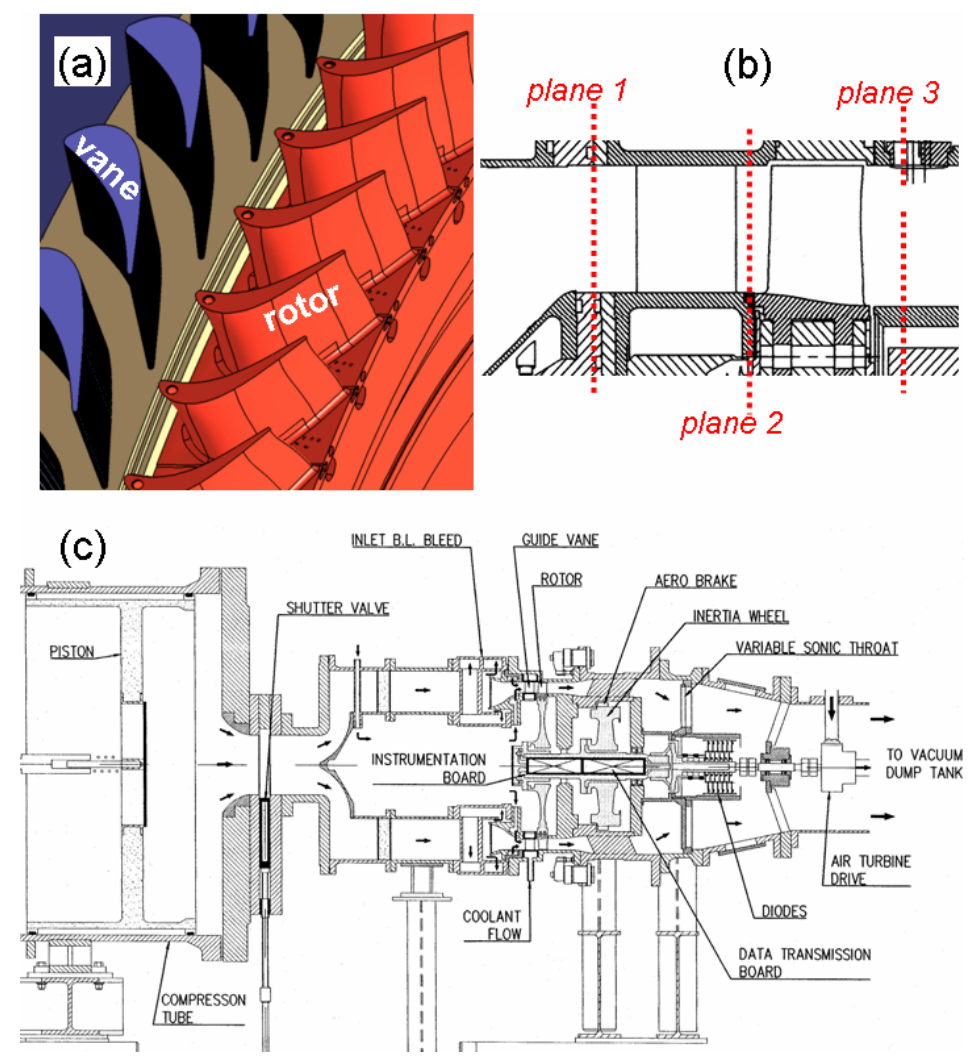

Fig. 9: a) Compression tube rig used for turbine testing. b) Meridional and 3-D view of the investigated turbine stage.

\section{Phase-locking}

Downstream of the turbine rotor, the flow follows a periodic evolution at the blade passing frequency. For this reason, the flow behavior is described best by retrieving the aerodynamic variables from the Phase-Locked Averaged (PLA) pressures, illustrated in Fig. 10. These are computed by isolating a number of blade passages, dividing them into a fixed number of "classes", and making averages among the corresponding classes (..i-1, i, i+1 ...). The number of classes is chosen as the number of samples recorded per blade passing event. The evolution of the flow is then plotted as a function of the "phase". The zero phase $\left(t / T_{r}=0\right)$ is defined as the position in which the stacking axis of the blades is directly in front of the sensor. The phase is equal to 1 after the rotor has completed one rotor pitch displacement. The probe sensors are not located at the same angular position, hence the pressure traces are shifted in phase to the axis of the probe. 

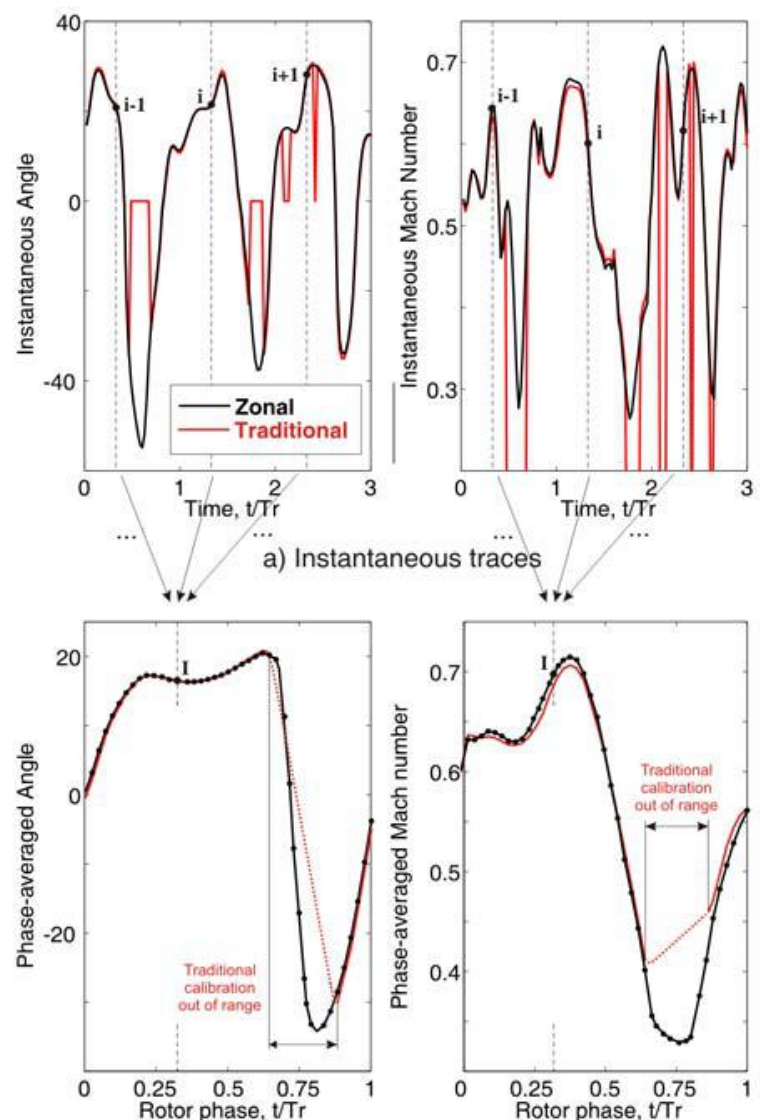

b) Phase-averaged distributions

Fig. 10: a) Instantaneous flow variables obtained with the traditional calibration and with the zone-based data reduction method. b) Phase-locked averaging procedure.

Fig. 10-a shows the flow angle and the local Mach number from a measurement in the tip region (87.3\% span), downstream of the transonic turbine stage. In regions where the traditional calibration is out of range, the novel method provides a reading. Fig. 10-b shows the resulting phase averaged pitch-wise distribution of the flow angle and the Mach number. Again, the zonal calibration allows to retrieve the angle and Mach number over the whole phase, unlike the traditional method.

\section{Time-averaged turbine exit flow measurements}

Fig. 11 represents the radial distribution of the time-averaged outlet flow angle. The experimental data is compared with the NISRE results (Non-Isentropic Radial Equilibrium) to help identifying the secondary and tip leakage flows. As the pressure ratio increases, the Mach number raises. Since the rotational speed is constant for all conditions the mean outlet flow angle increases with the pressure ratio, $-20 \mathrm{deg}$. at Low and $+25 \mathrm{deg}$. at High. Close to hub, between 10 and $20 \%$ of the span, an overturning core is identified; this is probably due to the rotor hub 
passage vortex. Close to the tip ( $\sim 90 \%$ of the span) a clear underturning region appears, that is related to the rotor tip leakage.

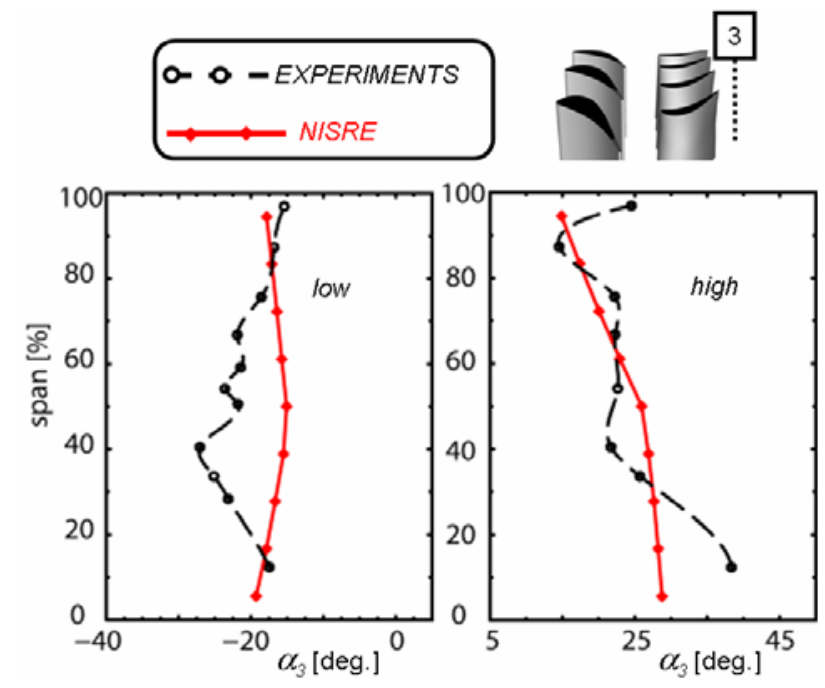

Fig. 11: Time-averaged flow angle downstream of the turbine at Low and High conditions (in red, results of the Non Isentropic Radial Equilibrium model of the turbine).

\section{Unsteady flow measurements}

The PLA of the outlet flow angle, total pressure and absolute Mach number are shown in Fig. 12. Large angular fluctuations are observed across a pitch at $86 \%$ span $(16 \mathrm{deg}$. in the Low condition, and $40 \mathrm{deg}$. In the High condition). These large angular fluctuations are caused by the tip clearance vortex which is driven by a jet of hot gas that leaks from the pressure side towards the suction side of the rotor blade, mostly in the trailing edge region (Heider et al, 1993). The underturning associated to the passage vortex is identified at about $25 \%$ of the span.

At High large fluctuations of total pressure $\left(P_{03}\right)$ are observed at phase 0.6, corresponding to the passing of the rotor blades trailing edge shock.

The passage of the rotor trailing edge shocks is made clearer in Fig. 13. The rapid decrease of Mach number from supersonic to subsonic levels, together with the high levels of random unsteadiness evidence the passage of a rotor locked shock pattern, sweeping the probe in a periodic manner, as sketched in Fig. 13-c. 


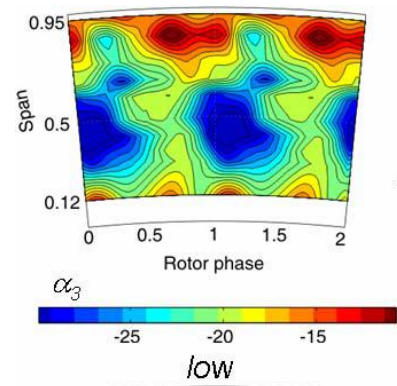

(a)
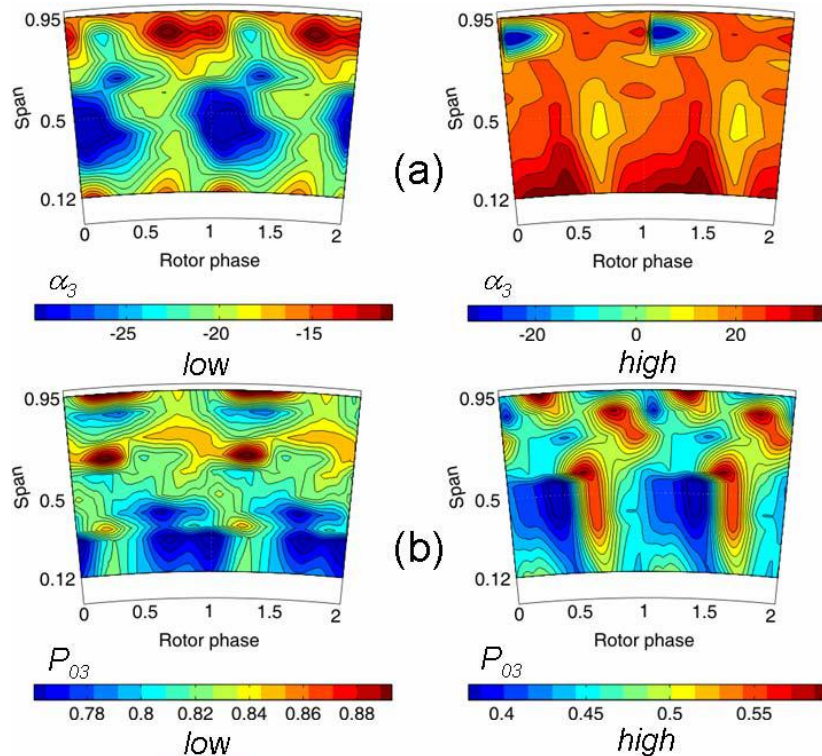

(b)
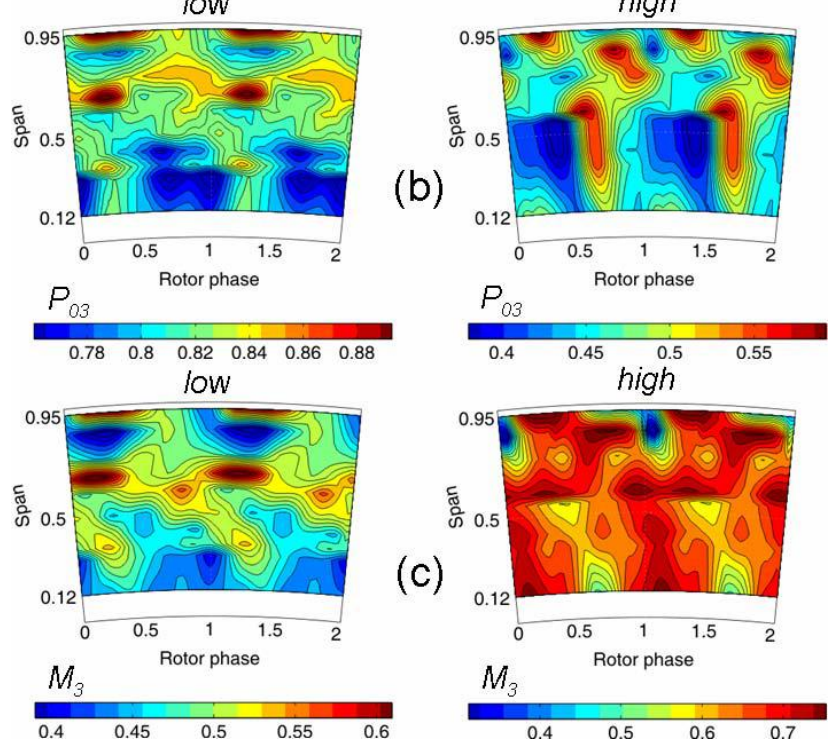

Fig. 12: Time-resolved rotor outlet flow field: a) Angle distribution; b) Total pressure contour; c) Absolute Mach number.
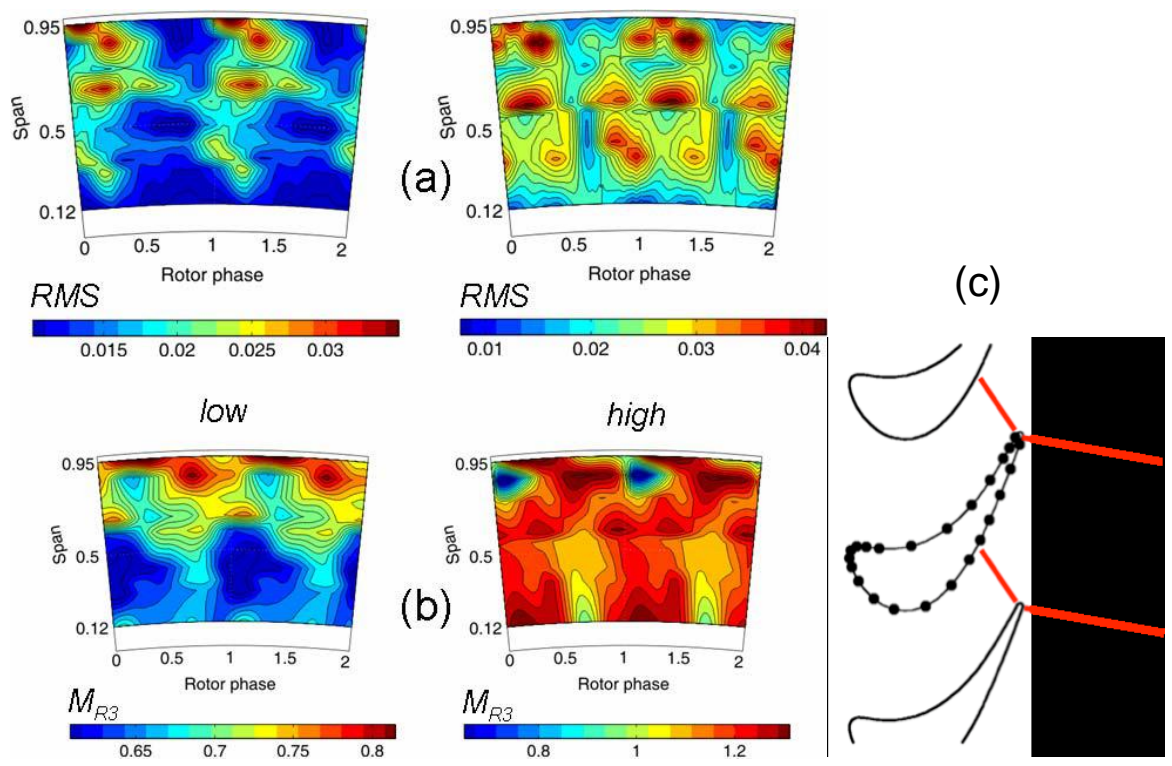

Fig. 13: a) Random unsteadiness for the low and high condition. b) Rotor relative outlet Mach number 


\section{Conclusions}

Fast response aerodynamic probes have become an essential tool in the study of the flow field in turbomachines thanks to their ability to measure both the steady and unsteady components of the flow vector as well as the total and static pressures.

The accuracy of the measurements depends on many different factors. The requirements of the turbine test rig have led to the choice of a wedge shaped probe head with a $60 \mathrm{deg}$. apex angle: it exhibits a large calibration range with good dynamic characteristics. Indeed, unsteady errors are reduced by the absence of cavity resonance and the limited effect of vortex interaction downstream of the probe. Still, both an extensive angular calibration and a careful sensor calibration are needed. At this point, the application of a zonal calibration technique allowed preserving the whole angular range and assuring in-range measurements even when large flow fluctuations are expected. An analysis of the impact of the uncertainty on the pressure sensor readings on the retrieved variables was also conducted for the zonal technique, revealing a low uncertainty for the whole angular range in all Mach conditions.

Numerical simulations showed that the large separation at the rear of the probe had little influence on the flow over the lateral sensors, and that the recirculation bubble remains of small size from 0 to $20 \mathrm{deg}$. yaw angle. Also, the numerical results indicate that the calibration at atmospheric pressure is valid for the turbine measurements, roughly at half the static pressure.

The present study has also shown how the combination of the VKI wedge probe with a novel zonal calibration method has provided steady and unsteady measurements downstream of the turbine stage, and has allowed to identify secondary flows and the presence of shocks downstream of the stage. Designers can benefit from a better understanding of unsteady flow physics and achieve improvement in the turbine design. Furthermore, the contribution of this work to the knowledge in the behavior of fast response probes will facilitate the development of future measurement tools.

\section{Acknowledgements}

The authors wish to thank the contributions of T. Yasa, G. Persico, and B. Adiloglu for their advice and support during the measurement campaign. The authors would like to acknowledge the financial support of the European Commission and European manufacturers that are participating to the Project TATEF2 "Turbine Aero-Thermal External Flows 2". 


\section{REFERENCES}

Ainsworth R. W., Miller R. J., Moss R. W., Thorpe S. J., 2000, "Unsteady pressure measurement", Measurement Science and Technology, Vol. 11, pp 1055-1076.

Ainsworth R.W., Allen J.L., Batt J.J.M., 1995, “The Development of Fast Response Aerodynamic Probes for Flow Measurements in Turbomachinery”, Journal of Turbomachinery. Vol. 117, pp 625-634.

Ainsworth R.W., Dietz A.J., Nunn T.A., 1991, “The Use of Semiconductor Sensors for Blade Surface Pressure Measurements", Journal of Engineering for Gas Turbines and Power.Vol. 113, pp 159.

Albrecht H.-E., Damaschke N., Borys M., Tropea C., 2003, "Laser Doppler and Phase Doppler Measurement Techniques". 738 pp, Springer. ISBN: 978-3-540-67838-0

Argüelles Díaz, K.M., Fernández Oro, J.M., Blanco, E., 2008, “Direct calibration framework of triple-hole pressure probes for incompressible flow", Measurement Science and Technology, Vol. 19, 075401 (13pp).

Bohn D. and Schnittfeld Th., 1992, "The dynamic response of capillary tubes for use in miniature pressure probes". Proceedings of the $11^{\text {th }}$ Symposium on "Measuring Techniques for Transonic and Supersonic Flow in Cascades and Turbomachines”. München, Germany.

Brouckaert J.F., Sieverding C.H., Manna M., 1998, "Development of fast response 3-hole pressure probe". Proceedings of the $14^{\text {th }}$ Symposium on Measuring Techniques for Transonic and Supersonic Flows in Cascades and Turbomachines. Limerick, Ireland.

Brunn H.H., 1995, “Hot-wire Anemometry”, Oxford University Press, N.Y.

Bubeck H., and Wachter J., 1987, "Development and application of a high frequency wedge probe". Proceedings of ASME Turbo Expo. ASME Paper 87-GT-216.

Cherret M.P., 1992, “Temperature error compensation applied to pressure measurements taken with miniature semi-conductors pressure transducers in a high speed research compressor". Proceedings of the $11^{\text {th }}$ Symposium on Measuring Techniques for Transonic and Supersonic Flow in Cascades and Turbomachines. München. Germany.

Cook S.C.P., 1989, “The Development of a High Response Aerodynamic Wedge Probe and Use on HighSpeed Research Compressor", Proceedings of the $9^{\text {th }}$ Symposium on Air Breathing Engines. ISABE 89-7118. Athens, Greece.

Dénos R., 2002, "Influence of Temperature Transients and Centrifugal Force on Fast Response Pressure Transducers". Experiments in fluids. Volume 33, Issue 2, pp 256-264.

Dibelius G., and Minten G., 1983, "Measurements of unsteady pressure fluctuations using capillary tubes". Proceedings of the $7^{\text {th }}$ Symposium on "Measuring Techniques for Transonic and Supersonic Flow in Cascades and Turbomachines". Aachen, Germany.

Dominy R.G., Hodson H.P., 1992, “An investigation of factors influencing the calibration of 5-hole probes for 3-D flow measurement”. Proceedings of ASME Turbo Expo. ASME Paper 92-GT 216.

Dudzinski T.J. and Krause L.N., 1969, "Flow direction measurement with fixed position probes". NASA Technical memorandum. NASA TM X-1904. October.

Epstein A.H., 1985, “ High frequency response measurements in turbomachinery Measurement Techniques in Turbomachines”. von Karman Institute Lecture Series. 1985-03. Rhode-Saint-Genese, Belgium.

Gossweiler C., Humm H.J., Kupferschmied P., 1990, “The use of piezoresistive pressure transducers for fast response pressure measurements in turbomachinery", Proceedings of the $10^{\text {th }}$ Symposium on Measuring Techniques for Transonic and Supersonic Flow in Cascades and Turbomachines, von Karman Institute. Belgium. 
Heider R., Duboué J.M., Petot B., Billonet G., Couiller V. and Liamis N., 1993, “Three-Dimensional Analysis of Turbine Rotor Flow Including Tip Clearance”. Proceedings of ASME Turbo Expo. ASME Paper 93-GT-111.

Hodson H.P., and Dambach R., 1999, "Single-sensor fast response pressure probes - the "least-squares" method of data reduction". Proceedings of the $14^{\text {th }}$ Symposium on Measuring Techniques in Transonic and Supersonic Flow in Cascades and Turbomachines, Limerick, Ireland.

Hufman G.D., Rabe D.C., Poti N.D., 1980, "Flow direction probes from a theoretical and experimental point of view”. Journal Phys. E: Sci. Instrum. Vol. 13, pp 751-760.

Humm H.J., Gossweiler C., Gyamarthy G., 1994, “On fast response probes: Part 2 - Aerodynamic probe design studies”. Proceedings of ASME Turbo Expo. ASME Paper 94-GT-27. The Hague, Netherlands.

Kerrebrock J.L., Epstein A.H., Haines D.M., Thompkins W.T., 1974, “The MIT Blowdown Compressor Facility", Journal of Engineering for Power, Vol. 46, No. 4.

Kline S.J. and McClintock, F.A., 1953, "Describing Uncertainties in Single Sample Experiments", ASME Mech. Eng., Vol. 75, pp 3-8

Krause L. N. and Dudzinski T. J., 1969, "Flow-direction Measurement with Fixed Position Probes in Subsonic Flow over a Range of Reynolds Numbers", Proceedings of the $15^{\text {th }}$ Int. ISA Aerospace Instrumentation Symposium. Las Vegas, USA.

Lou W., Aalburg C., Hourmouziadis J., 1998, "Total and static quantity measurements in unsteady flow Effect of unsteadiness". Proceedings of the ASME Turbo Expo. ASME Paper 98-GT-533.

Matsunaga S., Fukumoto R., Ichiyanagi K., 1975, "Characteristics of a Combined Probe Calibrated with Polynomials to Measure Unsteady Flows”. Bulletin of JSME. Vol.18, No. 122, pp. 834-840.

Paniagua G., Dénos R., 2002, "Digital compensation of pressure sensors in the time domain". Journal Experiments in Fluids. Vol. 32, No. 4, pp 417-424. DOI: 10.1007/s003480100355. ISSN: 0723-4864.

Paniagua G., Dénos R., Arts T., 2001, “Steady-unsteady measurements of the flow field downstream of a transonic HP turbine stage". Journal of Power and Energy. Vol. 215, No. A6, pp 663-673. DOI: 10.1243/0957650011538974. ISSN: 0957-6509.

Raffel, M., Willert, C.E., Wereley, S.T., Kompenhans, J., 2007, "Particle Image Velocimetry a Practical Guide". $2^{\text {nd }}$ edition, 448 pp, Springer. ISBN: 978-3-540-72307-3.

Redionitis K.O., and Chrysanthakopoulus G., 1998, "Application of nueral networks and fuzzy logic to the calibration of the seven hole probe". Journal of Fluids Engineering. Vol. 120, pp 95-101.

Reichert B.A. and Wendt B.J., 1994, "A new algorithm for five-hole probe calibration, data reduction and uncertainty analysis”. NASA Technical Memorandum 106458. Nasa Lewis Research Center. September.

Rodriguez O., 1984, "The circular cylinder in subsonic and transonic flow”, AIAA Journal, Vol. 22 , No 12.

Senoo Y., Kia Y., and Ookuma K., 1973, "Measurements of two dimensional periodic flow with a cobra probe". Journal of Fluids Engineering. June, pp 295.

Sieverding C.H., Arts T., 1992, “The von Karman Institute compression tube annular cascade facility CT-3”, Proceedings of the ASME Turbo Expo. ASME Paper 92-GT-336.

Sieverding C.H., Arts T., Denos R., and Brouckaert J.F., 2000, "Measurement techniques for unsteady flows in turbomachines". Experiments in Fluids. Vol. 28, No 4, pp 285-321.

White F.M., 2006, "Fluid Mechanics”. Ed. McGraw-Hill, $6^{\text {th }}$ edition, ISBN-10: 0072938447.

Zinn H., and Habermann M., 2007, "Developments and experiences with pulsation measurements for heavyduty gas turbines". Proceedings of ASME Turbo Expo. ASME Paper GT2007-27475. 
This document is a pre-print version of the scientific paper published by Springer. It has been released by the authors to fulfill all the publisher requirements established for Article Sharing:

https://www.springer.com/gp/open-access/authors-rights

\section{(9) $\Theta \Theta \Theta$}

(C) 2019. This manuscript version is made available under the Creative Commons Attribution-NonCommercial-NoDerivatives 4.0 International License (CC-BY-NC-ND 4.0 license) http://creativecommons.org/licenses/by-nc-nd/4.0/ 